\title{
MRI of Hip Cartilage
}

\section{Joint Morphology, Structure, and Composition}

\author{
Stephanie L. Gold BA, Alissa J. Burge MD, \\ Hollis G. Potter MD
}

Published online: 22 June 2012

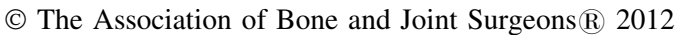

\begin{abstract}
Background Accurate, reproducible, and noninvasive assessment of hip cartilage is clinically relevant and provides a means by which to assess the suitability of candidates for arthroscopic or open surgical procedures and the response to such interventions over time. Given the relatively thin cartilage of the hip and the complex spherical anatomy, however, accurately assessing the cartilage poses a challenge for traditional MRI techniques.

Questions/Purposes We assessed the current status of imaging articular cartilage of the hip through a comprehensive review of recent literature.

Methods We performed a literature review using PubMed. Topics included quantitative MRI, imaging of the hip cartilage and labrum, femoroacetabular impingement syndrome, and osteoarthritis of the hip.

Where Are We Now? With the use of high in-plane and through-plane resolution, reproducible assessment of hip cartilage and labrum is clinically feasible. More recent quantitative MR techniques also allow for noninvasive
\end{abstract}

The institution of the authors has received during the study period research support from GE Healthcare (Princeton, NJ, USA).

All ICMJE Conflict of Interest Forms for authors and Clinical Orthopaedics and Related Research editors and board members are on file with the publication and can be viewed on request. This work was performed at Hospital for Special Surgery, New York, NY, USA.

S. L. Gold, A. J. Burge, H. G. Potter ( $₫)$

Department of Radiology and Imaging, Hospital for Special Surgery, 535 East 70th Street, New York, NY 10021, USA

e-mail: potterh@hss.edu

H. G. Potter

Weill Cornell Medical College of Cornell University,

New York, NY, USA assessment of collagen orientation and proteoglycan content in articular cartilage, thus providing insight into early matrix degeneration. These techniques can be applied to cohorts at risk for osteoarthritis, helping to predict cartilage degeneration before symptoms progress and osteoarthritic changes are visible on radiographs.

Where Do We Need to Go? Prospective longitudinal data registries are necessary for developing predictive models of osteoarthritis and subsequent joint failure to assess the results of surgical intervention and predict the timing of arthroplasty.

How Do We Get There? By establishing more hip cartilage registries, a correlation can be made between subjective measures and morphologic MRI to assess the cartilage, labrum, bone, and synovial lining of the hip.

\section{Introduction}

Patients commonly present with generalized hip pain in the presence of normal radiographs, posing a diagnostic dilemma for physicians. The etiology of mechanical hip pain can be either intraarticular or extraarticular, leading to an incredibly diverse list of differential diagnoses [7]. Some of the most common causes of hip and groin pain include osteoarthritis, labral tears, avascular necrosis, and fracture. Synovial proliferative diseases, snapping hip syndrome, and greater trochanteric pain syndrome can also produce hip pain. Chondral and labral pathology is commonly associated with disorders such as femoroacetabular impingement (FAI), developmental dysplasia (DDH), and slipped capital femoral epiphysis (SCFE), all of which can result in early osteoarthritis.

Given the recent increase in the number of hip arthroscopy procedures performed, studies have documented correlations 
between the results of preoperative MRI findings and intraoperative inspection of the joint [7]. The current status review of peer-reviewed literature demonstrates accurate assessment of cartilage can be achieved using high-resolution noncontrast or MR arthrographic techniques, the latter of which are obtained with the intraarticular insulation of dilute gadolinium compounds [20]. The advent of quantitative MR has provided a noninvasive means by which to assess articular cartilage biochemistry, and preliminary studies have applied these metrics to cohorts at risk for osteoarthritis [16, 21]. Assessment of hip cartilage biochemistry is essential to provide a more sensitive means by which to assess early osteoarthritis that may occur before development of morphologic changes perceived on standardized MR techniques or before the development of osteoarthritic changes on standardized radiographs.

In this review, we present the current state of MRI of the hip, including (1) conventional morphologic imaging techniques, (2) newer quantitative MRI techniques to assess tissue biochemistry, and (3) the application of these imaging techniques for cohorts at risk for osteoarthritis. We also discuss the optimal implementation of these techniques to assess the risk for osteoarthritis and the potential strategies for achieving multiinstitutional registries.

\section{Search Strategy and Criteria}

We performed a literature review using PubMed limited to articles published after 1997 (given the more rapid advancement in MRI techniques after this time) and those published in the English language (given restrictions in translational services). Topics included quantitative MRI, imaging of the hip cartilage and labrum, FAI syndrome, and osteoarthritis of the hip. Roughly 60 articles were initially referenced and 28 were used for the final publication.

\section{Where Are We Now? Conventional Imaging Techniques}

For best results, clinical hip MRI should be performed on a 1.5- (Table 1) or 3-T (Table 2) magnet, utilizing a two-part shoulder coil, small-field-of-view wrap coil, or multiplechannel cardiac coil. The phased-array torso coil is commonly used to image the hip, as it can increase the signal-to-noise ratio while maintaining visualization of both hips [27]. Moderate echo time fast spin echo (FSE) techniques with an effective echo time of 34 milliseconds at $1.5 \mathrm{~T}$ and 28 milliseconds at $3 \mathrm{~T}$ [24] yield optimal differential tissue contrast.

Coronal images provide optimal evaluation of the superior labrum, suprafoveal femoral head articular cartilage, and the cartilage over the lateral acetabular dome. The hip abductors, short external rotators, and the iliopsoas attachments are optimally evaluated in this plane. In addition, the abductor origins and the iliofemoral ligament are visible. Sagittal images provide optimal evaluation of the anterior labrum, where the majority of labral tears occur in the absence of a history of posterior subluxation [12]. In addition, the articular cartilage of the femoral head and acetabular dome can be evaluated. Axial images allow visualization of the anterior and posterior acetabular articular cartilage and labrum. The iliopsoas tendon and bursa are also well visualized, as are the sciatic, femoral,

Table 1. Imaging parameters of the hip at $1.5 \mathrm{~T}$ (coil: body, phased-array shoulder; position: feet first supine)

\begin{tabular}{|c|c|c|c|c|c|}
\hline Parameter & $\begin{array}{l}\text { Coronal fast inversion } \\
\text { recovery (whole pelvis) }\end{array}$ & $\begin{array}{l}\text { Axial FSE } \\
\text { (whole pelvis) }\end{array}$ & $\begin{array}{l}\text { Sagittal FSE } \\
\text { (affected hip) }\end{array}$ & $\begin{array}{l}\text { Coronal FSE } \\
\text { (affected hip) }\end{array}$ & $\begin{array}{l}\text { Oblique axial FSE } \\
\text { (affected hip) }\end{array}$ \\
\hline TR (ms) & $3500-5000$ & $4000-5000$ & $4000-5000$ & $4000-5000$ & $4000-5000$ \\
\hline $\mathrm{TE}(\mathrm{ms})$ & 17 & 34 & 34 & 34 & 34 \\
\hline TI & 150 & & & & \\
\hline BW (Hz/pixel) & 200 & 200 & 200 & 200 & 200 \\
\hline ETL & $7-9$ & $8-12$ & $9-12$ & $9-12$ & $9-12$ \\
\hline NEX & 2 & 2 & 3 & 3 & 3 \\
\hline FOV $(\mathrm{cm})$ & $\begin{array}{l}\text { Greater intertrochanteric } \\
\text { distance plus } 2 \mathrm{~cm}\end{array}$ & $\begin{array}{l}\text { Greater } \\
\quad \text { intertrochanteric } \\
\text { distance }\end{array}$ & $17-18$ & $17-18$ & 16 \\
\hline Matrix & $256 \times 192$ & $512 \times 256$ & $512 \times 384$ & $512 \times 384$ & $512 \times 256$ \\
\hline Slice/Gap (mm) & $5 / 0$ & $5 / 0$ & $2.5 / 0$ & $4 / 0$ & $3 / 0$ \\
\hline NPW & Yes & Yes & Yes & Yes & Yes \\
\hline Frequency & $\mathrm{R} / \mathrm{L}$ & $\mathrm{A} / \mathrm{P}$ & $\mathrm{A} / \mathrm{P}$ & $\mathrm{R} / \mathrm{L}$ & $\mathrm{A} / \mathrm{P}$ \\
\hline
\end{tabular}

$\mathrm{TR}=$ repetition time; $\mathrm{TE}=$ echo time; $\mathrm{TI}=$ time to inversion; $\mathrm{BW}=$ receiver bandwidth; $\mathrm{ETL}=$ echo train length; $\mathrm{NEX}=$ number of excitations; FOV = field of view; $\mathrm{NPW}=$ no phase wrap; $\mathrm{FSE}=$ fast $\operatorname{spin}$ echo; $\mathrm{R} / \mathrm{L}=$ right to left; $\mathrm{A} / \mathrm{P}=$ anterior to posterior. 
Table 2. Imaging parameters of the hip at $3.0 \mathrm{~T}$ (coil: cardiac; position: feet first supine)

\begin{tabular}{|c|c|c|c|c|c|}
\hline Parameter & $\begin{array}{l}\text { Coronal fast inversion } \\
\text { recovery (whole pelvis) }\end{array}$ & $\begin{array}{l}\text { Axial FSE } \\
\text { (whole pelvis) }\end{array}$ & $\begin{array}{l}\text { Sagittal FSE } \\
\text { (affected hip) }\end{array}$ & $\begin{array}{l}\text { Coronal FSE } \\
\text { (affected hip) }\end{array}$ & $\begin{array}{l}\text { Oblique axial FSE } \\
\text { (affected hip) }\end{array}$ \\
\hline TR (ms) & $4000-5000$ & 2500 & $3500-5000$ & $4000-5000$ & 4000 \\
\hline $\mathrm{TE}(\mathrm{ms})$ & 16 & 27 & 26 & 34 & 27 \\
\hline TI & 190 & & & & \\
\hline BW (Hz/pixel) & 250 & 250 & 250 & 250 & 250 \\
\hline ETL & $12-18$ & $8-12$ & 12 & 18 & 12 \\
\hline NEX & 1 & 2 & 2 & 2 & 2 \\
\hline FOV $(\mathrm{cm})$ & $\begin{array}{l}\text { Greater intertrochanteric } \\
\text { distance plus } 2 \mathrm{~cm}\end{array}$ & $\begin{array}{l}\text { Greater } \\
\text { intertrochanteric } \\
\text { distance }\end{array}$ & $17-18$ & $17-18$ & $17-18$ \\
\hline Matrix & $288 \times 192$ & $512 \times 256-320$ & $512 \times 384-416$ & $512 \times 384-416$ & $512 \times 320-416$ \\
\hline Slice/Gap (mm) & $5 / 0$ & $5 / 0$ & $2-2.5 / 0$ & $3-3.5 / 0$ & $3 / 0$ \\
\hline NPW & Yes & Yes & Yes & Yes & Yes \\
\hline Frequency & $\mathrm{R} / \mathrm{L}$ & $\mathrm{A} / \mathrm{P}$ & $\mathrm{A} / \mathrm{P}$ & $\mathrm{R} / \mathrm{L}$ & $\mathrm{A} / \mathrm{P}$ \\
\hline
\end{tabular}

$\mathrm{TR}=$ repetition time; $\mathrm{TE}=$ echo time; $\mathrm{TI}=$ time to inversion; $\mathrm{BW}=$ receiver bandwidth; $\mathrm{ETL}=$ echo train length; $\mathrm{NEX}=$ number of excitations; FOV = field of view; $\mathrm{NPW}=$ no phase wrap; $\mathrm{FSE}=$ fast $\operatorname{spin}$ echo; $\mathrm{R} / \mathrm{L}=$ right to left; $\mathrm{A} / \mathrm{P}=$ anterior to posterior.

Fig. 1A-B (A) Coronal and (B) sagittal surface coil FSE images of the left hip in a 57-year-old woman with mild dysplasia demonstrate actively delaminating cartilage yielding exposed bone over the femoral head and acetabular dome, with chondral flap formation at 12:00 (arrows).
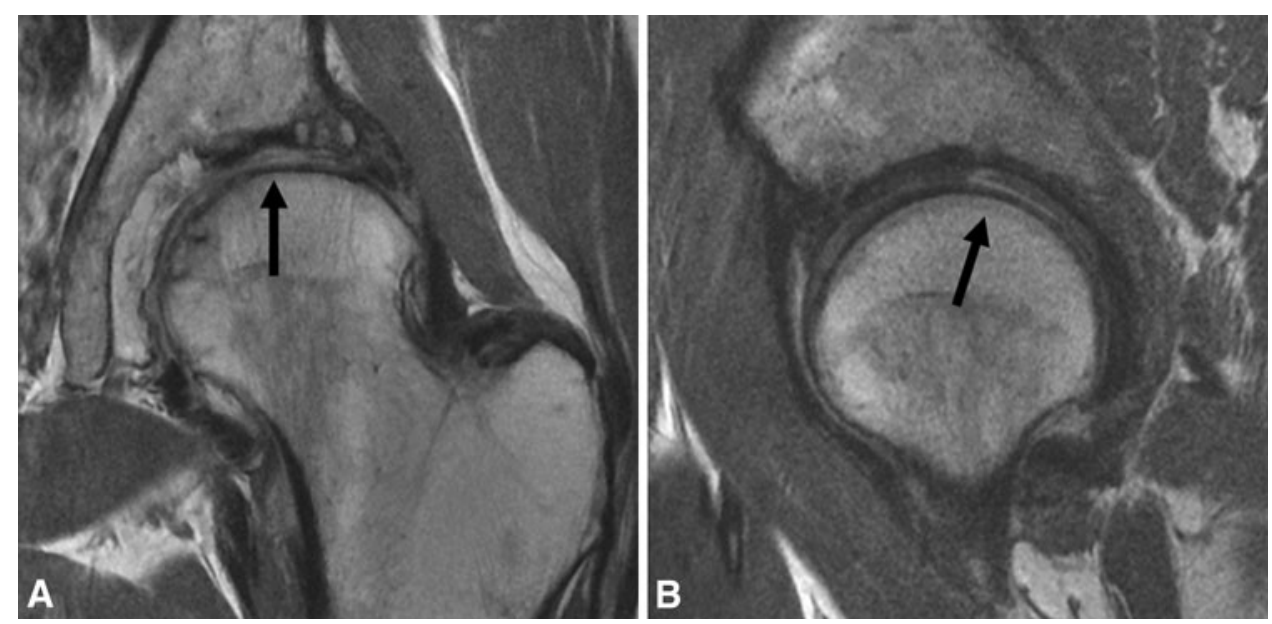

and obturator nerves. The bare area should be assessed on axial images for the presence of loose bodies or tears of the ligamentum teres in the presence of microinstability. Axial oblique images oriented along the axis of the femoral neck are commonly utilized in the evaluation of FAI syndrome. Axial images of the distal femoral condyles are commonly obtained for hip version analysis [24]. Large-field-of-view fat-suppressed images of the entire pelvis are useful for identifying the presence of edema and fluid collections and for detecting intrapelvic pathology, such as tumors.

Imaging of the Cartilage and Labrum

Cartilage, a metabolically active tissue made up of water, proteoglycan, and collagen, has unique MR signal characteristics. Traditional T1-weighted three-dimensional fatsuppressed gradient echo sequences allow for the differentiation between low-signal-intensity bone and highsignal-intensity cartilage but are less sensitive for partialthickness defects and result in substantial artifact in the postoperative setting. An intermediate-weighted twodimensional non-fat-suppressed FSE sequence is often favored, providing reduced scan time, accurate assessment of partial-thickness chondral defects, and good contrast between the bone, cartilage, and joint fluid [24]. MRI can demonstrate full-thickness cartilage defects, progressive degeneration, and fibrillation in the presence of preserved joint space on radiographs. Actively delaminating cartilage manifests as a hyperintense signal adjacent to the bonecartilage interface, where the water should be most restricted (Fig. 1). MRI can also distinguish chondral shear from osteochondral fracture, the latter of which is amenable to primary bioabsorbable pin fixation [26] (Fig. 2). Osteochondral lesions may also be noted, and considerations for MRI include not only the status of the articular 
cartilage but also the viability of the subchondral bone and potential stability of the lesion (Fig. 3).

The fibrocartilaginous labrum appears as a low-signalintensity triangular structure attached to the acetabular margin. While abnormalities of the labrum are usually defined by changes in shape and signal intensity, Aydingoz and Ozturk [1] showed the appearance of the labrum varied with age and sex. Among the 180 asymptomatic patients

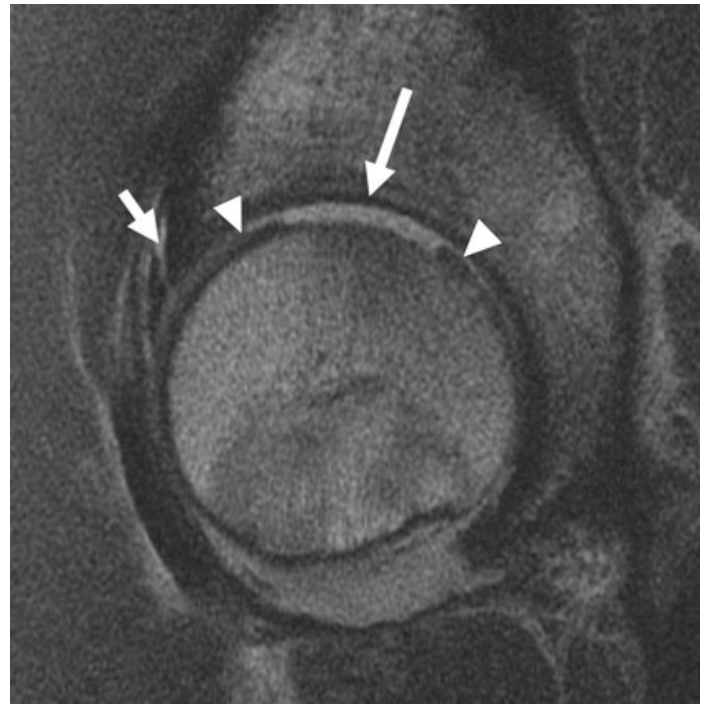

Fig. 2 A sagittal surface coil FSE image of the right hip in a 25-yearold man with a history of posterior hip dislocation demonstrates traumatic chondral shear involving the femoral head from the 11:00 to 2:00 positions (long arrow), as well as an anterior labral tear (short arrow). Note the intact intermediate signal intensity cartilage at the anterior and posterior margins of the defect (arrowheads). examined, the labrums varied considerably in shape: $69.2 \%$ were triangular, $15.8 \%$ were round, $12.5 \%$ appeared flat, and $2.5 \%$ were not visible [1]. Given this variation, MRI findings of the labrum should always be correlated with the clinical presentation.

Tears of the labrum often coexist with lesions of the articular cartilage. Labral tears can be caused by traumatic injury, degenerative disease, or capsular laxity. The majority of labral tears affect the anterior aspect of the labrum and the less common posterior labral tears may be caused by posterior instability. On MRI, labral tears are indicated by an increased signal intensity with fluid signal between the labrum and acetabulum [27]. Labral tears are often associated with the development of paralabral cysts, which can lead to secondary osseous erosion.

MR arthrography is often thought to be superior to conventional noncontrast techniques for evaluating the cartilage and labrum. Typical MR arthrography sequences to evaluate the cartilage include fat-suppressed T1-weighted FSE images in all three planes. Additional fluid-sensitive images should be acquired. Given the recent increase in the number of hip arthroscopies performed each year, correlation between MRI and intraoperative findings is possible. Using standard MR arthrography protocols, Blankenbaker et al. [8] demonstrated a sensitivity of $70 \%$ and a specificity of $84 \%$ in detecting cartilage defects identified at arthroscopy. That study compared traditional arthrography sequences with iterative decomposition of water and fat with echo asymmetry and least-squares estimation with spoiled gradient echo (IDEAL-SPGR), demonstrating in the detection of cartilage lesions IDEAL-SPGR had a lower $(\mathrm{p}<0.001)$
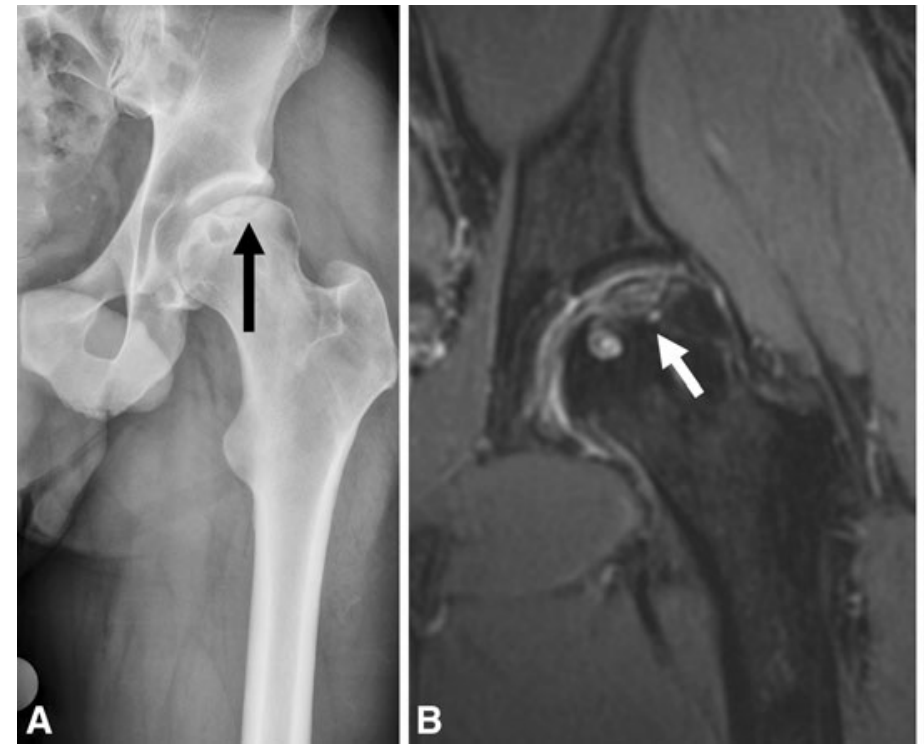

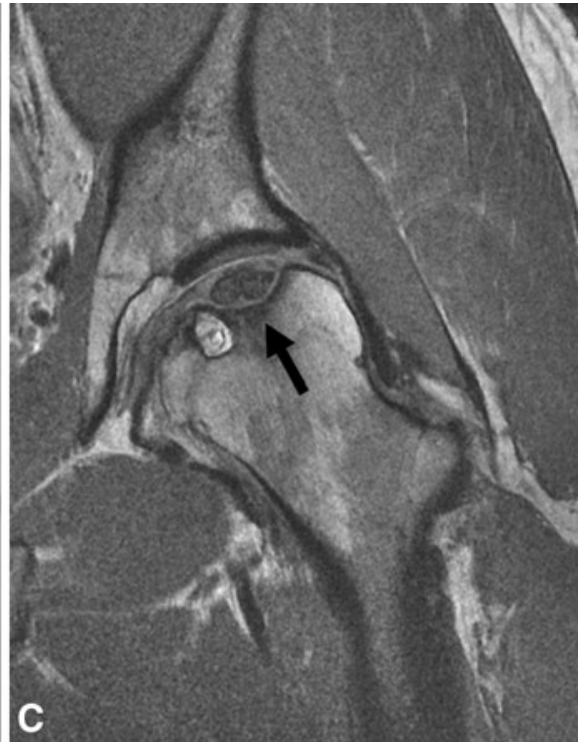

mature bed, manifesting as sclerosis surrounding the lesion, which contains devitalized fragments with overlying abnormal, hyperintense cartilage (arrows). 
specificity [8]. Focusing on patients with FAI, Schmid et al. [25] evaluated the diagnostic performance of MR arthrography in detecting cartilage and labral abnormalities. In all regions combined, the sensitivity and specificity of detecting surgically confirmed cartilage lesions were $79 \%$ and $77 \%$, respectively, for the first reader and $50 \%$ and $84 \%$, respectively, for the second reader [25]. In a more recent study, Byrd and Jones [9] compared the diagnostic accuracy of noncontrast MRI with MR arthrography in evaluating the articular cartilage of the hip. In detecting labral tears, this study found noncontrast MRI had a sensitivity of $25 \%$ and a specificity of $65 \%$ while MR arthrography has a sensitivity of $66 \%$ and a specificity of $75 \%$. In evaluating chondral lesions, MRI had a specificity of $18 \%$ and a specificity of $100 \%$ while MR arthrography had a sensitivity of $41 \%$ and a specificity of $100 \%$ [9]. In those studies with reduced accuracy, careful review of both in-plane and through-plane (slice thickness) resolution often reveals thicker slices and larger pixel size, both of which may act to decrease lesion conspicuity.

While MR arthrography has been advocated for the evaluation of labral pathology, this technique requires intraarticular administration of gadolinium-based contrast, a procedure that is not without risks. Noncontrast MRI of the hip, utilizing an optimized protocol, is capable of identifying labral pathology without the need for intraarticular contrast. Mintz et al. [20] demonstrated detection of labral pathology using arthroscopy as the standard; 84 of 88 labral tears were correctly identified, with a sensitivity of $97 \%$ and accuracy of $93 \%$. In the same study, an accuracy of $87 \%$ to $88 \%$ and weighted-kappa measures of reproducibility of $0.8(\mathrm{p}<0.001)$ were achieved for articular cartilage over the dome and femoral head, demonstrating reproducible and accurate assessment of the cartilage is possible without the increased risk and cost of intraarticular contrast [20]. The decision to image using either MR arthrography or high-resolution noncontrast techniques should be based on the personal experience of the referring clinician and his/her MR radiologist, comparing the preoperative imaging to findings at arthroscopy.

\section{Where Are We Now? Advanced Quantitative Cartilage Imaging Techniques}

Quantitative MR techniques are increasingly being used to noninvasively assess collagen orientation and proteoglycan content in articular cartilage to detect early osteoarthritis before the development of morphologic changes on standardized MRI or radiographs. Charged gadolinium contrast agents, T1 $\rho$, sodium MRI, T2 relaxation, and cartilage volume/thickness mapping are all powerful tools used to complement conventional morphologic images [13]. These techniques show great promise in providing predictive models of osteoarthritis and helping to predict time of joint failure.

\section{T2 Mapping}

Direct calculation of the transverse relaxation time constant, T2, can provide quantitative information regarding the collagen orientation of cartilage. This technique involves the acquisition of several images per slice, each at a different echo time. Calculating the slope of the T2 decay curve allows for determination of $\mathrm{T} 2$ on a pixel-by-pixel basis. T2 values are color coded and mapped onto a grayscale image. The resulting $\mathrm{T} 2$ map provides a visual assessment of water content and collagen fiber orientation; in the deep zone of cartilage near the subchondral plate, the relaxation times are shorter due to the high order of the collagen in the radial zone. More prolonged values are found in the transitional zone, closer to the articular surface. Alterations in the expected zonal stratification allow recognition of areas of early osteoarthritis, sometimes with morphologically normal cartilage [14, 17] (Fig. 4).

\section{$\mathrm{T} 1 \rho$}

Spin lattice relaxation in the rotating frame, or $\mathrm{T} 1 \rho$, is a cartilage-mapping technique that discerns regional differences in proteoglycan content of native cartilage without the use of intravenous contrast. Given the laminar architecture of cartilage and the differential distribution of proteoglycan within each layer, T1 $\rho$ allows one to discern areas of abnormal proteoglycan content corresponding to foci of cartilage degeneration. Elevated T1 $\rho$ values reflect areas of proteoglycan depletion [17]. The utility of this technique in detecting early cartilage degeneration has been documented at clinical field strengths [10, 15] (Fig. 4).

\section{Delayed Gadolinium-Enhanced MRI of Cartilage}

Delayed gadolinium-enhanced MRI of cartilage (dGEMRIC) is an imaging technique that relies on the differential distribution of a negatively charged diethylenetriaminepentaacetic acid gadolinium(III)dihydrogen salt (Gd-DTPA) within articular cartilage. The differential distribution comes from the interaction between negatively charged glycosaminoglycans (GAGs) and negatively charged Gd-DTPA salt, which results in contrast distribution inversely proportional to the amount of GAG present. Cartilage degeneration results in depletion of proteoglycan, resulting in increased uptake of gadolinium as compared to normal cartilage. This technique requires the intravenous administration of a relatively large 

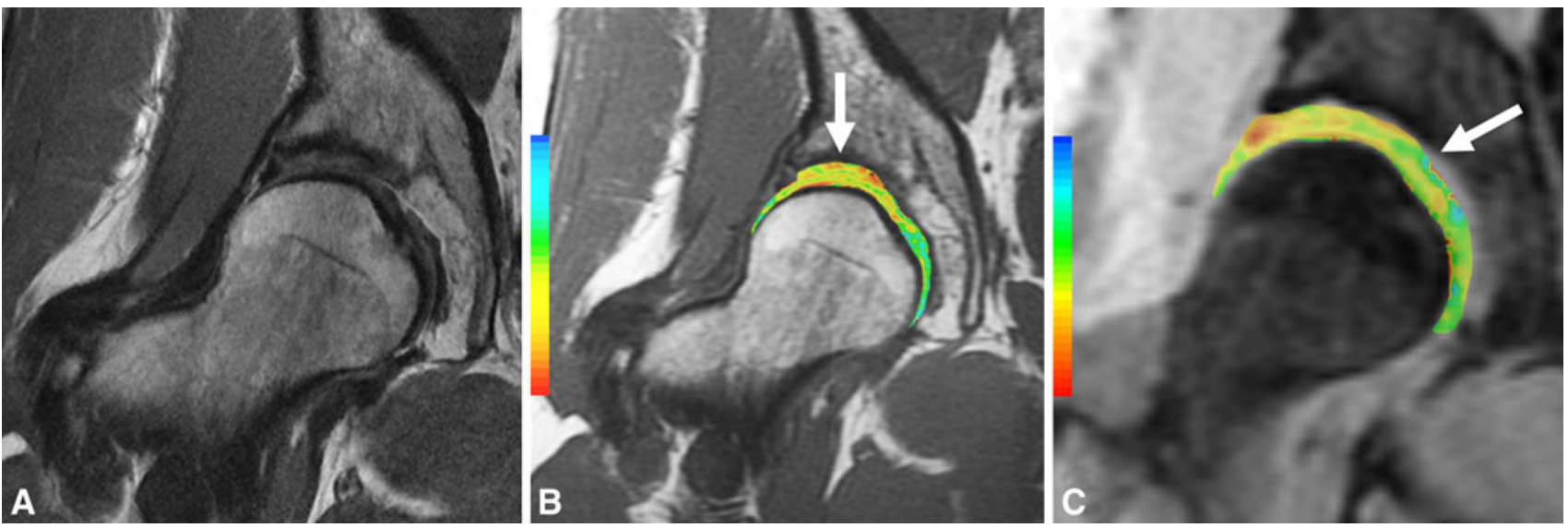

Fig. 4A-C (A) Coronal FSE, (B) T2 map, and (C) T1 $\rho$ images demonstrate preservation of cartilage morphology in a 42-year-old woman with DDH being considered for periacetabular osteotomy; note the prolongation of $\mathrm{T} 2$ and $\mathrm{T} 1 \rho$ values at the suprafoveal region of the femoral head and the superior aspect of the dome (arrows).

Fig. 5A-B (A) Coronal and (B) axial surface coil FSE images of the right hip in a 31-year-old woman with DDH demonstrate a characteristically shallow acetabulum with uncovering of the lateral aspect of the femoral head resulting in compensatory labral hypertrophy (arrows) and chondral wear.
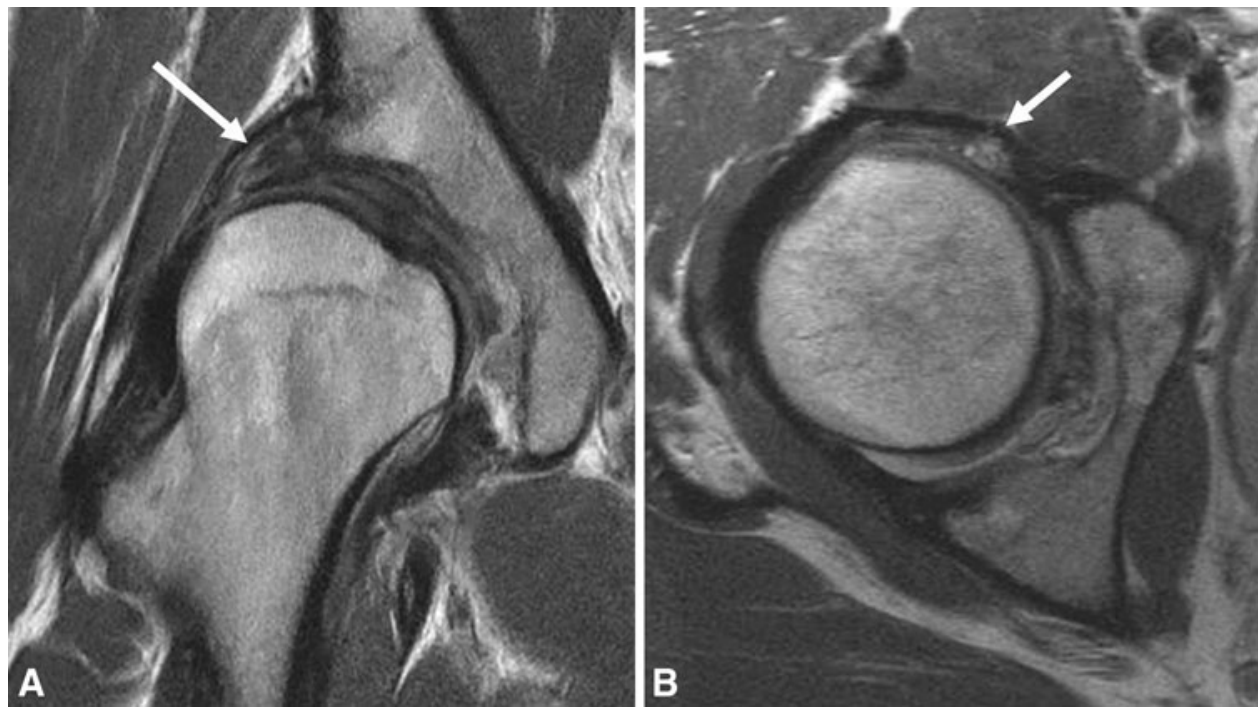

amount of gadolinium, followed by a 15-minute course of light exercise to disperse the gadolinium; imaging is typically performed 45 to 90 minutes after injection, although some suggest a shorter time may be adequate $[2,14]$.

Quantitative MRI shows great promise for providing predictive models of osteoarthritis with the hope of predicting time of joint failure. More data are needed from prospective studies to achieve this aim.

\section{Where Are We Now? Imaging Cohorts at Risk for Osteoarthritis}

\section{DDH and SCFE}

In adults with DDH, MRI shows a characteristically shallow fovea and acetabular dome, with cartilage loss and hypertrophy of the anterior and superior labrum (Fig. 5).
Early signs of cartilage degeneration on MRI include chondral hyperintensity and loss of normal grayscale stratification. Surface fibrillation may also be noted, which is commonly seen before more substantial cartilage loss. Quantitative MR techniques are helpful for determining the degree of cartilage loss and can aid in identifying patients suitable for periacetabular osteotomy versus those who require arthroplasty [24]. Kim et al. [16] showed dGEMRIC is a sensitive measure for the development of early osteoarthritis in patients with DDH. This group also showed dGEMRIC was the most important predictor of failure of periacetabular osteotomy [11]. Finally, T2 mapping of the hip has been used to detect early osteoarthritis in patients with DDH [21]. In patients with clinically suspected SCFE, MRI can demonstrate the angular deformity at the metaphysic, the status of the cartilage, and any signs of osteonecrosis. MRI is also useful in assessing the physeal closure after surgical treatment and the 
development of any postoperative complications such as avascular necrosis or secondary FAI, even in the presence of instrumentation [6] (Fig. 6).

\section{FAI Syndrome}

While radiographs are generally used to diagnose FAI, MRI is useful for evaluating the articular cartilage and labrum, detecting early features of osteoarthritis when

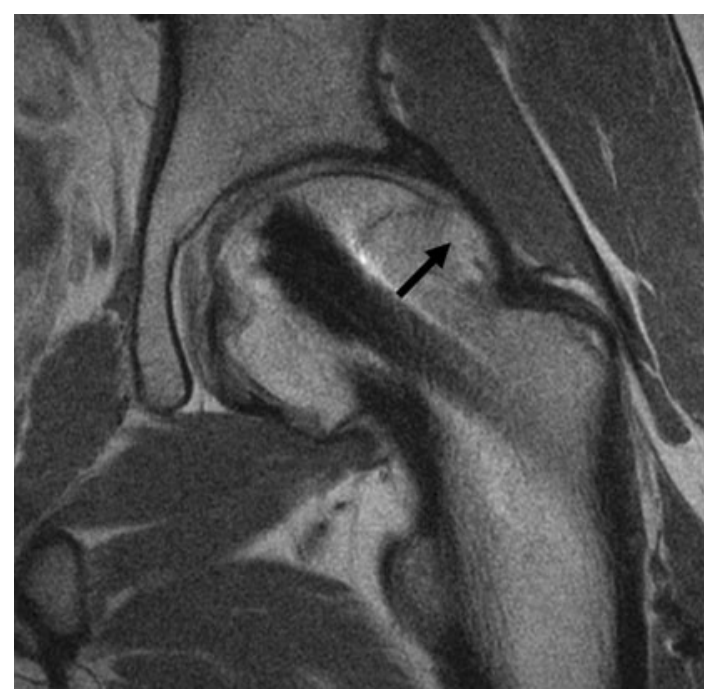

Fig. 6 A coronal surface coil FSE image of the left hip in a 17-yearold adolescent boy with indwelling instrumentation for SCFE demonstrates secondary FAI, with marked insufficient offset and extensive proliferative bone formation at the anterolateral femoral head-neck junction (arrow). radiographs are negative. The oblique axial view is used to define and measure the insufficient offset at the head-neck junction. It is also helpful for measuring the alpha angle, a quantitative measure of the prominence of the anterior head-neck junction. Notzli et al. [22] studied the use of the alpha angle and determined angles of $50^{\circ}$ or greater are suggestive of cam impingement. However, pincer impingement is typically characterized by normal alpha angles [3]. While some investigators have questioned the variability in alpha angle measurements [18], with consistent and careful attention to measuring techniques (sometimes requiring radial acquisitions to define the point of maximum offset deficiency), the alpha angle can be used as a reproducible diagnostic tool [4]. Three-dimensional CT may also be helpful in discerning the bony anatomy in FAI and specialized software may be used to predict the increase in flexion possible from an arthroscopic neck débridement [5].

MRI specifically of cam-type impingement shows insufficient head-neck offset and rapid cartilage loss over the anterosuperior margin of the dome. Active delamination may be seen, manifesting as hyperintensity in the basilar component of the cartilage over the anterior dome [24] (Fig. 7). Evaluation of the femoral head will demonstrate an osseous deformity at the anterolateral aspect of the femoral head-neck junction, often with intraosseous ganglion cysts adjacent to the anterior capsule and thickening of the iliofemoral ligament. Regional scarring of the synovial lining may also be present. MRI of pure pincer impingement demonstrates intralabral ossification at the anterior margin of the joint and the lateral margin of the dome, as well as anterosuperior impingement against
Fig. 7A-B (A) Coronal and (B) sagittal FSE images demonstrate insufficient offset of the head-neck junction with proliferative bone formation and delaminating cartilage over the anterior margin of the dome (arrow) in a 42-year-old man with cam FAI.
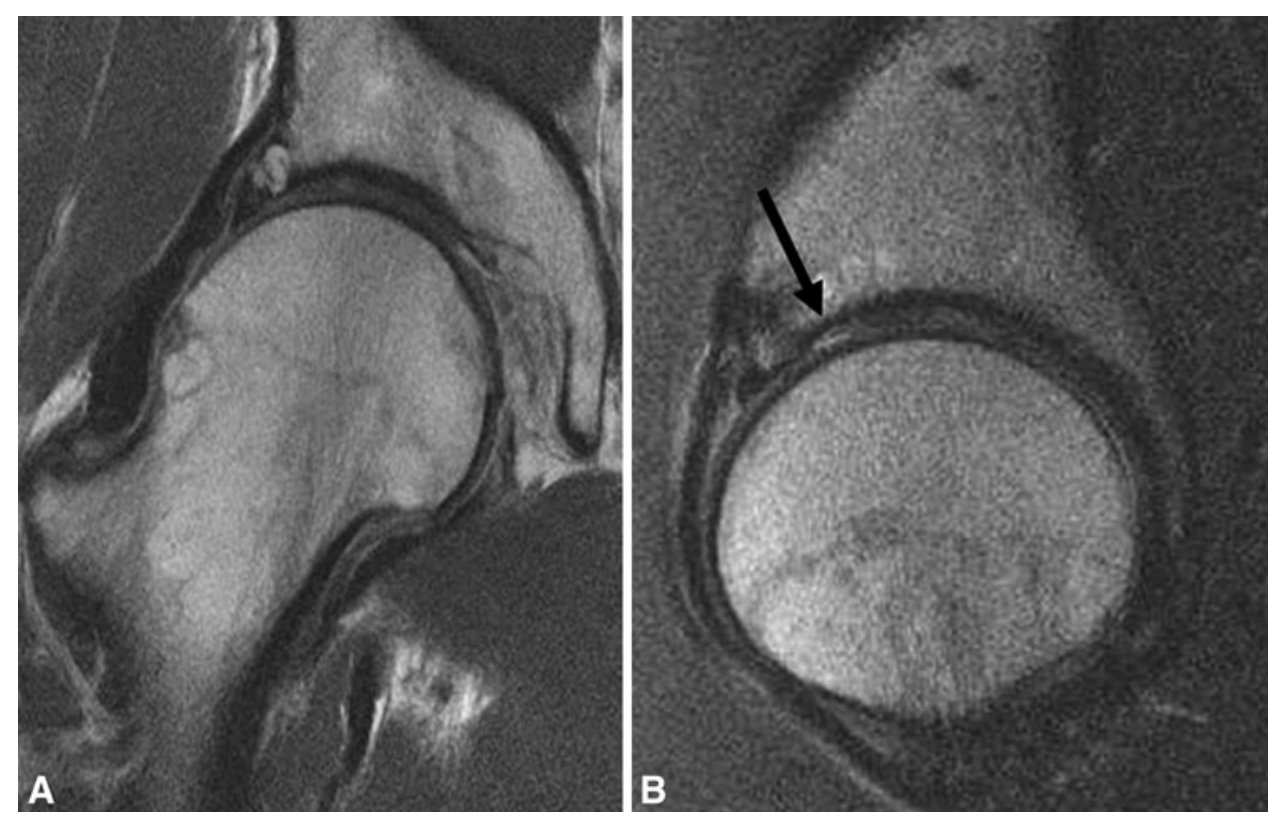
Fig. 8A-B (A) Coronal and (B) axial surface coil FSE images of the left hip in a 28-year-old woman demonstrate minimal proliferative bone formation at the anterior aspect of the femoral neck (black arrow) and endosteal callus formation along the compressive margin of the femoral neck (white arrow).
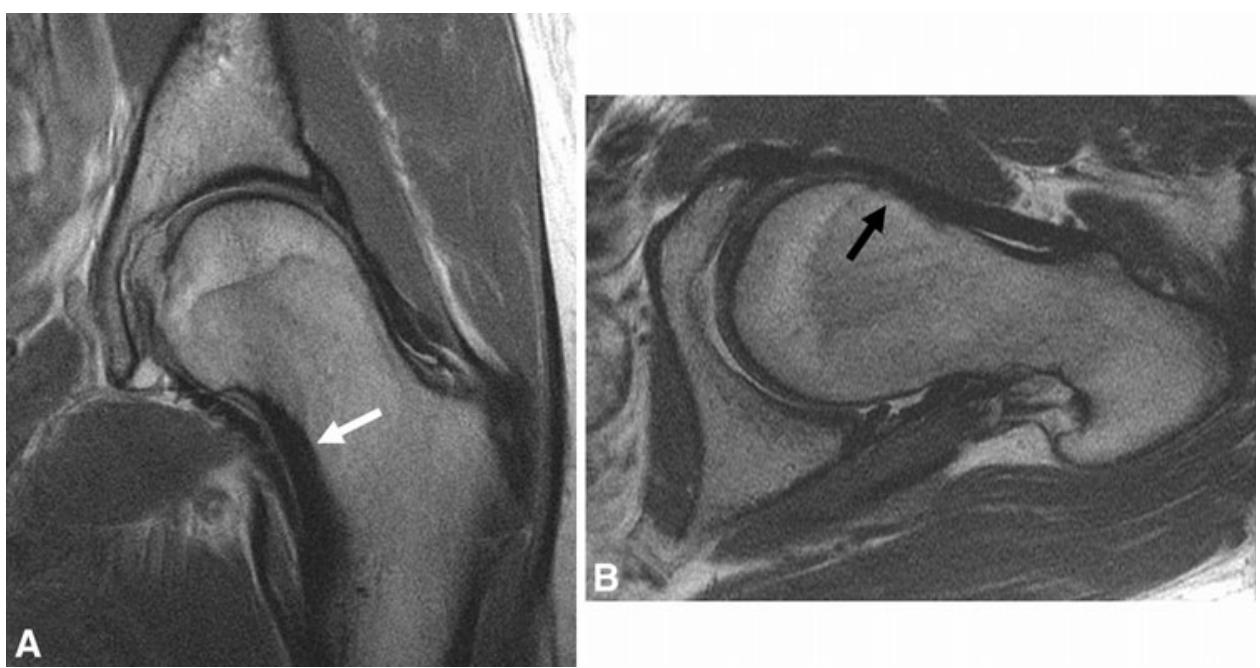

the proximal medial acetabular rim [28]. Frank coxa profunda and acetabular retroversion may also be seen [27]. Pincer impingement manifests with primary labral failure followed by slower, progressive cartilage loss.

It is clear many patterns of FAI exist, often with subtle imaging differences. While males more typically present with the classic appearance of proliferative bone formation, FAI in females is often associated with less prominent cam lesions; indeed, neck sclerosis or subtle periosteal bone formation may be the only features noted (Fig. 8). FAI in females is also seen with coxa valga or DDH, thus confounding surgical decision making, as treatment of these conditions are quite different. Careful review of the radiographs and MRI, combined with a thorough clinical examination, will result in a definitive treatment.

In the postoperative setting, MRI can assess the degree of neck débridement and may be used as a noninvasive tool by which to assess the progression of osteoarthritis. Complications may also be detected, such as insufficiency fractures of the femoral neck, particularly in females (Fig. 9), as well as postsurgical deficiencies of the iliofemoral ligament and capsule, which may be implicated in instability patterns (Fig. 10). After labral repair, the presence of fluid imbibition into the repair site or a displaced fragment is most predictive of failure to heal or retear, respectively (Fig. 11).

Despite the recent enthusiasm for the use of MRI to diagnose FAI, some argue caution is needed in making diagnostic decisions based on imaging findings [23]. Moreover, it has been suggested impingement syndromes exist in roughly $10 \%$ to $15 \%$ of the population, but symptomatic osteoarthritis is far less common. Therefore, criteria need to be established to help determine which patients would benefit from surgical intervention and MRI is extremely well suited to aid in this distinction [27].

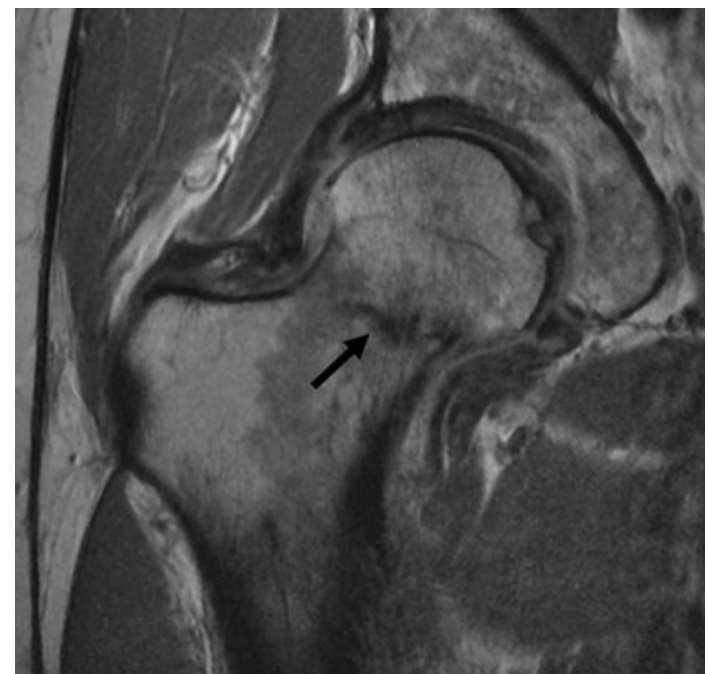

Fig. 9 A coronal surface coil FSE image of the right hip in a 51-yearold woman 1 month after femoral neck débridement for treatment of FAI demonstrates a compressive-side basicervical stress fracture (arrow) of the femoral neck with extension to the tensile side.

\section{Discussion}

Chronic generalized hip pain is commonly seen in clinical practice and the number of hip arthroscopies performed each year is expected to double by 2013 [19]. Given this, we presented the current state of MRI of the hip, including both conventional morphologic imaging techniques and newer quantitative techniques to evaluate the cartilage. Applying these techniques to cohorts at risk for osteoarthritis will provide a means of noninvasively assessing the biochemistry and morphology of cartilage, which can help guide clinical management.

The literature and our review are subject to a number of limitations. First, only articles in the English language were used, as translation services were limited. Second, only 
Fig. 10A-B (A) Coronal and (B) axial FSE images demonstrate disruption of the iliofemoral ligament (arrows) in a 24-year-old man with a history of prior arthroscopic femoral neck débridement for treatment of FAI with development of postoperative instability.
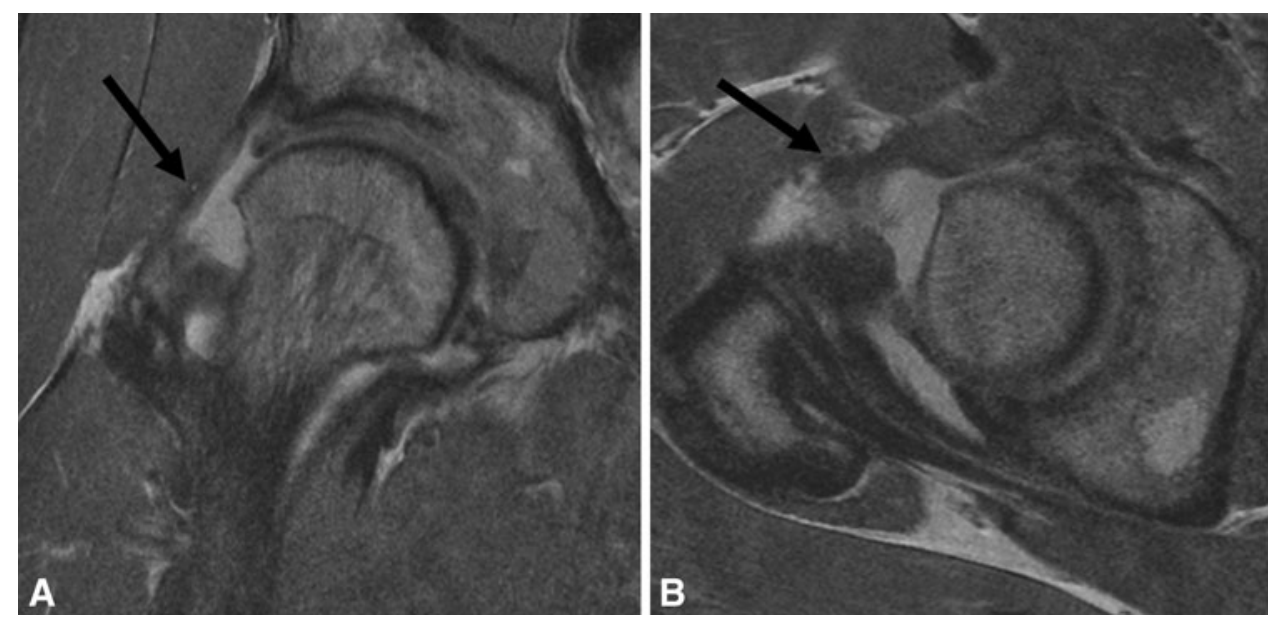

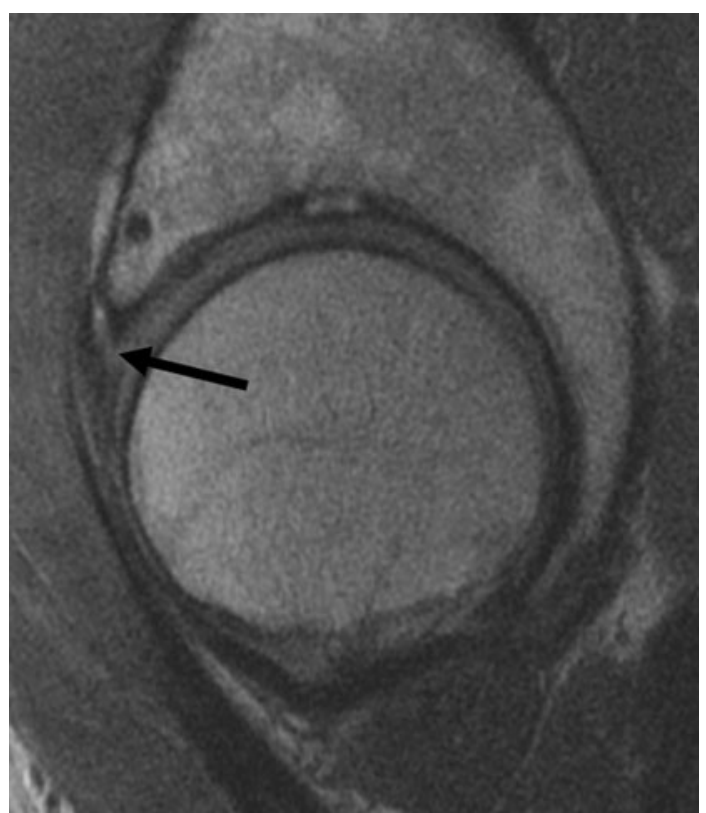

Fig. 11 A sagittal surface coil FSE image of the right hip in a 42-year-old woman with a history of prior labral repair demonstrates labral retear (arrow), with a split through the base of the anterior labral remnant.

publications before 1997 were referenced, given the fact that MRI of cartilage is an incredibly dynamic field and major advancements have been made in the past decade. Third, it is definitely possible that studies are in press and thus not available for review. This process limits the conclusions that may be drawn from this review; in particular, the lack of prospective, longitudinal data sets limits our ability to develop predictive hypotheses about the rate of progression of cartilage morphologic change or depletion of matrix, as denoted by quantitative MRI.

Currently, both high-resolution noncontrast MRI and MR arthrography provide reproducible, accurate assessment of hip cartilage, labrum, bone, and synovial lining.
FSE techniques using a moderate echo time on either a 1.5- or 3-T magnet yield optimal tissue contrast for imaging the hip. An intermediate-weighted, water-sensitive two-dimensional non-fat-suppressed gradient echo is useful for imaging the cartilage and labrum, accurately detecting partial-thickness chondral defects. While some consider MR arthrography to be superior to conventional MRI for evaluation of labral pathology, our institution has demonstrated, using an optimized protocol, reproducible and accurate assessment of the cartilage and labrum is possible without the increased risks of intraarticular contrast. Controversy still exists regarding the optimal means by which to assess the articular cartilage of the hip for regional morphologic change. By comparing the preoperative imaging with findings at arthroscopy, radiologists can determine whether MR arthrography is necessary to accurately detect cartilage and labral pathology.

Quantitative MR techniques such as dGEMRIC, T2 mapping, and $\mathrm{T} 1 \rho$ can detect changes in collagen orientation and proteoglycan content, providing insight into early matrix degeneration. This biochemical assessment of the hip cartilage provides noninvasive information that can detect early osteoarthritic changes that may be occult on conventional MR images and radiographs.

High-resolution noncontrast MRI and newer quantitative techniques can be used to identify early signs of cartilage degeneration. These techniques are perhaps most helpful in cohorts at risk for developing early osteoarthritis, such as those with FAI, DDH, or SCFE. In patients with early osteoarthritis and minimal joint damage, surgery to correct the bony deformity can improve symptoms; however, more longitudinal data are needed to determine whether these procedures delay the natural progression of osteoarthritis.

More longitudinal data from hip registries are needed to build predictive models of joint failure, assess the results of surgical interventions aimed at delaying the progression of osteoarthritis, and help to predict the timing of arthroplasty. 
Multiinstitutional research efforts should correlate MRI assessment of cartilage morphology, labral integrity, and the synovial lining, using a standardized cartilage scoring system, with quantitative MR techniques that assess both proteoglycan content (dGEMRIC, T1 $\rho$ ) and collagen orientation (T2 mapping).

Longitudinal assessment using quantitative MRI will allow for the establishment of quantitative threshold values of the dGEMRIC index, $\mathrm{T} 2$ relaxation time, or $\mathrm{T} 1 \rho$ that are predictive of osteoarthritis and joint degradation that require arthroplasty. These studies should be focused on cohorts at risk for the development of early osteoarthritis, such as those with FAI or DDH. The imaging data can then be correlated to standard radiographic assessment and subjective clinical outcome instruments of pain and function. Given the latter instruments may be affected by extraarticular pathology, additional assessment of the adjacent soft tissues, such as the hip abductors, is necessary. Without the addition of objective imaging of cartilage and synovium as an independent outcome measure in longitudinal registries, the information is limited to the subjective outcome instruments of pain and function rather than the direct assessment of cartilage biology. Further, these measures will enable a noninvasive means by which to assess response to treatment, be it surgical or pharmaceutical. For example, does neck débridement for FAI actually slow the rate of cartilage matrix depletion compared to unoperated controls, as assessed by T1 $\rho$ and T2 mapping? Does intraarticular viscosupplementation affect cartilage homeostasis and matrix production? The correlations derived from such longitudinal data could then be used to develop predictive models of osteoarthritis and the factors that dictate a more rapid progression to joint arthroplasty, ultimately helping to identify patients who would benefit from early intervention and guide clinical management.

\section{References}

1. Aydingoz U, Ozturk MH. MR imaging of the acetabular labrum: a comparative study of both hips in 180 asymptomatic volunteers. Eur Radiol. 2001;11:567-574.

2. Bashir A, Gray ML, Boutin RD, Burstein D. Glycosaminoglycan in articular cartilage: in vivo assessment with delayed Gd(DTPA)(2-)-enhanced MR imaging. Radiology. 1997;205:551558.

3. Beall DP, Sweet CF, Martin HD, Lastine CL, Grayson DE, Ly JQ, Fish JR. Imaging findings of femoroacetabular impingement syndrome. Skeletal Radiol. 2005;34:691-701.

4. Beaule PE, Rakhra K. Cam-type FAI: is the alpha angle the best MR arthrography has to offer? (Skeletal Radiol 2009;38(9):855862). Skeletal Radiol. 2010;39:201-202.

5. Bedi A, Dolan M, Hetsroni I, Magennis E, Lipman J, Buly R, Kelly BT. Surgical treatment of femoroacetabular impingement improves hip kinematics: a computer-assisted model. Am J Sports Med. 2011;39(suppl):43S-49S.
6. Berquist TH. Pelvis, hips and thigh. In: MRI of the Musculoskeletal System. Philadelphia, PA: Lippincott Williams \& Wilkins; 2001.

7. Blankenbaker DG, Tuite MJ. MR imaging of early hip joint degeneration. Magn Reson Imaging Clin N Am. 2011;19:365378.

8. Blankenbaker DG, Ullrick SR, Kijowski R, Davis KW, De Smet AA, Shinki K, Munoz del Rio A, Keene JS. MR arthrography of the hip: comparison of IDEAL-SPGR volume sequence to standard MR sequences in the detection and grading of cartilage lesions. Radiology. 2011;261:863-871.

9. Byrd JW, Jones KS. Diagnostic accuracy of clinical assessment, magnetic resonance imaging, magnetic resonance arthrography, and intra-articular injection in hip arthroscopy patients. Am J Sports Med. 2004;32:1668-1674.

10. Carballido-Gamio J, Bauer JS, Stahl R, Lee KY, Krause S, Link TM, Majumdar S. Inter-subject comparison of MRI knee cartilage thickness. Med Image Anal. 2008;12:120-135.

11. Cunningham T, Jessel R, Zurakowski D, Millis MB, Kim YJ. Delayed gadolinium-enhanced magnetic resonance imaging of cartilage to predict early failure of Bernese periacetabular osteotomy for hip dysplasia. J Bone Joint Surg Am. 2006;88:15401548.

12. Farjo L, Glick JM, Sampson TG. Hip arthroscopy for acetabular labral tears. Arthroscopy. 1999;15:132-137.

13. Gold GE, Chen CA, Koo S, Hargreaves BA, Bangerter NK. Recent advances in MRI of articular cartilage. AJR Am J Roentgenol. 2009;193:628-638.

14. Jazrawi LM, Alaia MJ, Chang G, Fitzgerald EF, Recht MP. Advances in magnetic resonance imaging of articular cartilage. J Am Acad Orthop Surg. 2011;19:420-429.

15. Keenan KE, Besier TF, Pauly JM, Han E, Rosenberg J, Smith RL, Delp SL, Beaupre GS, Gold GE. Prediction of glycosaminoglycan content in human cartilage by age, T1rho and T2 MRI. Osteoarthritis Cartilage. 2011;19:171-179.

16. Kim YJ, Jaramillo D, Millis MB, Gray ML, Burstein D. Assessment of early osteoarthritis in hip dysplasia with delayed gadolinium-enhanced magnetic resonance imaging of cartilage. J Bone Joint Surg Am. 2003;85:1987-1992.

17. Koff MF, Potter HG. Noncontrast MR techniques and imaging of cartilage. Radiol Clin North Am. 2009;47:495-504.

18. Lohan DG, Seeger LL, Motamedi K, Hame S, Sayre J. Cam-type femoral-acetabular impingement: is the alpha angle the best MR arthrography has to offer? Skeletal Radiol. 2009;38:855-862.

19. Matsuda D. Hip arthroscopy: update, advances, and future applications. AAOS Now. 2011;5:8.

20. Mintz DN, Hooper T, Connell D, Buly R, Padgett DE, Potter HG. Magnetic resonance imaging of the hip: detection of labral and chondral abnormalities using noncontrast imaging. Arthroscopy. 2005;21:385-393.

21. Nishii T, Tanaka H, Sugano N, Sakai T, Hananouchi $T$, Yoshikawa $\mathrm{H}$. Evaluation of cartilage matrix disorders by $\mathrm{T} 2$ relaxation time in patients with hip dysplasia. Osteoarthritis Cartilage. 2008;16:227-233.

22. Notzli HP, Wyss TF, Stoecklin CH, Schmid MR, Treiber K, Hodler J. The contour of the femoral head-neck junction as a predictor for the risk of anterior impingement. J Bone Joint Surg Br. 2002;84:556-560.

23. Palmer WE. Femoroacetabular impingement: caution is warranted in making imaging-based assumptions and diagnoses. Radiology. 2010;257:4-7.

24. Potter HG, Schachar J. High resolution noncontrast MRI of the hip. J Magn Reson Imaging. 2010;31:268-278.

25. Schmid MR, Notzli HP, Zanetti M, Wyss TF, Hodler J. Cartilage lesions in the hip: diagnostic effectiveness of MR arthrography. Radiology. 2003;226:382-386. 
26. Shindle MK, Kelly BT, Moya LE, Mintz DN. The hip. In: Khanna AJ, ed. MRI for Orthopaedic Surgeons. New York, NY: Thieme; 2010.

27. Stoller DW, Sampson TG, Li AE, Bredella MA. The hip. In: Stoller DW, ed. Magnetic Resonance Imaging in Orthopaedics and Sports Medicine. $3^{\text {rd }}$ ed. Philadelphia, PA: Lippincott Williams \& WIlkins; 2007.

28. Tannast M, Siebenrock KA, Anderson SE. Femoroacetabular impingement: radiographic diagnosis - what the radiologist should know. AJR Am J Roentgenol. 2007;188:1540-1552. 